\title{
Role of electronic structure in photoassisted transport through atomic-sized contacts
}

\author{
J. K. Viljas ${ }^{1,2}$ and J. C. Cuevas ${ }^{3,1,2}$ \\ ${ }^{1}$ Institut für Theoretische Festkörperphysik, Universität Karlsruhe, D-76128 Karlsruhe, Germany \\ ${ }^{2}$ Forschungszentrum Karlsruhe, Institut für Nanotechnologie, D-76021 Karlsruhe, Germany \\ ${ }^{3}$ Departamento de Física Teórica de la Materia Condensada, \\ Universidad Autónoma de Madrid, E-28049 Madrid, Spain
}

(Dated: February 6, 2008)

\begin{abstract}
We study theoretically quantum transport through laser-irradiated metallic atomic-sized contacts. The radiation field is treated classically, assuming its effect to be the generation of an ac voltage over the contact. We derive an expression for the dc current and compute the linear conductance in one-atom thick contacts as a function of the ac frequency, concentrating on the role played by electronic structure. In particular, we present results for three materials ( $\mathrm{Al}, \mathrm{Pt}$, and $\mathrm{Au}$ ) with very different electronic structures. It is shown that, depending on the frequency and the metal, the radiation can either enhance or diminish the conductance. This can be intuitively understood in terms of the energy dependence of the transmission of the contacts in the absence of radiation.
\end{abstract}

PACS numbers: 73.63.-b, 73.50.Pz, 73.63.Rt, 73.40.Jn

\section{INTRODUCTION}

The study of electronic transport in microscopic and nanoscale electrical contacts under the influence of timedependent external fields has a long history. Perhaps as the most famous example, superconducting tunnel junctions subjected to microwave radiation exhibit a step-like structure in their current-voltage $(I-V)$ characteristics [1]. This can be understood in terms of inelastic ("photoassisted") transport of electrons across the junction. In the early theoretical work of Tien and Gordon (TG) 2], this phenomenon was described by a harmonic voltage at the radiation frequency $\omega$ applied to one of the leads, giving rise to photo- "sidebands" associated with the "absorption" or "emission" of an integer multiple of the photon energy $\hbar \omega$. This theory has also been extended to describe superconducting atomic point contacts [3] and the predictions have been confirmed experimentally using microfabricated Al break-junctions under microwave irradiation [4]. In addition, a similar approach has been used to describe laser-irradiated junctions in scanning tunneling microscopes (STM) [5]. In these, as a result of inherent asymmetries in the geometry and the materials of the junction, laser irradiation can cause dc (rectification) currents even in the absence of a dc bias voltage [6, 7].

Over the years, several types of model calculations have been employed also to describe the ac-response of semiconductor heterostructures [8, 9] and other mesoscopic systems 10, 11, 12, as well as atomic and molecular contacts [13, 14, 15, 16, 17]. Among these, the TG-like theories have been quite successful in gaining a qualitative understanding of light-induced currents [1, 8, 13].

Metallic atomic-sized wires fabricated with STM or break-junction techniques have turned out to be ideal systems for investigating electronic transport at the nanoscale [18]. The bulk of the research in this field so far has concentrated on stationary transport properties, while systems being driven by time-varying external fields (such as laser light) have received less attention. Very recent experiments on laser-irradiated gold contacts support the idea that photoassisted processes may play an important role in their transport properties [19]. To take the first steps towards a microscopic description of experiments of this type, we address in this paper the role of electronic structure in photoassisted transport through atomic-sized junctions. This problem is not only relevant for the field of atomic contacts, but also for molecular electronics, where the role of the metallic contacts on photoassisted transport through molecular junctions remains to be understood.

In different types of metals, the nature and number of conduction channels in one-atom contacts reflect the valence of the metal, i.e., what type of atomic orbitals are available at the Fermi energy [20]. How is this difference between the metals seen in their response to irradiation? To investigate this question, we use a tight-binding model and explore mainly one-atom thick contact geometries like the one shown in Fig. 1(a). We are interested in the linear conductance $G_{d c}=\partial I /\left.\partial V\right|_{V=0}$ as a function of the radiation frequency $\omega$, which we call photoconductance. (a)

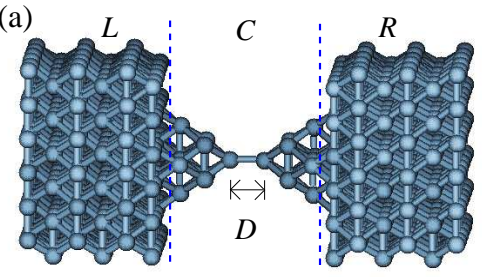

(b)

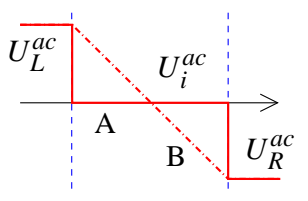

FIG. 1: (Color online) (a) Two infinite fcc [001] surfaces contacted with pyramids of 10 atoms in each, forming a "dimer" contact. The tip-to-tip distance is denoted with $D$, all other interatomic distances are the same as in bulk. (b) Two model ac voltage profiles $U_{i}^{a c}$, where $i$ labels atomic sites and orbitals in the $C$ region: a double step (A) and a linear ramp (B) between the lead values $U_{L}^{a c}$ and $U_{R}^{a c}$. 
Following the TG ideology, we model the effect of radiation with a time-periodic voltage, but we also take into account the effect of the voltage profile across the contact [see Fig. 1(b)]. While many previous model calculations with periodic driving fields are based on Floquet theory [8, 13], our method is based on non-equilibrium Green functions $[7,9,21,22]$. Our approach allows for a realistic description of the photoconductance of atomic contacts, with a single free parameter describing the local intensity of the radiation.

As examples of metals with very different electronic structures we choose $\mathrm{Al}, \mathrm{Pt}$, and $\mathrm{Au}$. We study one-atom thick junctions of these materials mainly in the so-called contact regime, but also in the tunneling regime. In the case of the contact regime we find the following results. For Pt the effect of the ac voltage is almost always to decrease $G_{d c}$ from its value in the absence of radiation. This is because the Fermi energy $\epsilon_{F}$ lies at the edge of the $d$ band, and exciting electrons above $\epsilon_{F}$ necessarily decreases their transmission probability, since less transmission channels are available there. For $\mathrm{Al}$, where $\epsilon_{F}$ is at the beginning of the $p$ band, also an enhancement of the conductance is possible. The magnitude of these changes depends on the intensity of the radiation, but can be up to $10 \%$ or more at visible frequencies. For $\mathrm{Au}, \epsilon_{F}$ is in the $s$ band, with a single completely open transmission channel for a wide range of energies. Thus, low-frequency radiation has no effect on the conductance, while in the visible range both an increase and a decrease are possible. These conclusions are based on detailed numerical simulations, but they can be understood in an appealing way in terms of the energy dependence of the transmission in the absence of the ac drive.

The rest of the paper is organized as follows. In Sec. II we introduce the theoretical model used to describe the electronic structure of atomic-sized contacts and the effect of irradiation. Section III is devoted to the derivation of a general formula for the dc current through atomic contacts subjected to an ac voltage. In Sec. IV we describe examples of numerical results of the frequency dependence of the linear conductance for one-atom thick contacts of Al, Pt, and Au. Finally, in Sec. $\mathrm{V}$ we discuss some of the assumptions and restrictions of our model and present the main conclusions.

\section{MODEL}

We describe the laser-irradiated point contact with an $s p d$-tight-binding Hamiltonian of the form

$$
\begin{gathered}
H(t)=H_{0}+H_{1}(t) \\
H_{0}=\sum_{i j} d_{i}^{\dagger} H_{i j} d_{j}, \quad H_{1}(t)=\sum_{i j} d_{i}^{\dagger} W_{i j}(t) d_{j},
\end{gathered}
$$

where the indices $i, j$ run over the different atoms and orbitals, including the two spin directions. The creation and annihilation operators $\left(d_{i}^{\dagger}\right.$ and $\left.d_{j}\right)$ satisfy $\left\{d_{i}, d_{j}\right\}=$
0 and $\left\{d_{i}, d_{j}^{\dagger}\right\}=\left[\boldsymbol{S}^{-1}\right]_{i j}$, where $\boldsymbol{S}$ is the overlap matrix of the non-orthogonal basis [23]. The Hamiltonian $H_{0}$ is for the system without radiation and dc voltage, while $H_{1}(t)$ includes them both. The matrix elements of the Hamiltonian and the overlap matrix $\left(H_{i j}\right.$ and $\left.S_{i j}\right)$ are taken from the parametrization of Ref. 24. All matrices in the spin-orbital basis (such as $\boldsymbol{H}$ and $\boldsymbol{S}$ ) are denoted with a boldface symbol.

We consider ideal symmetric geometries of the type shown in Fig. 1(a), with a single-atom thick constriction. This type of "dimer" structure is suggested by molecular dynamics simulations as the most common one in the last conductance plateau 25]. When the distance $D$ between the tip atoms corresponds to the bulk interatomic distance, the junction is said to be in the contact regime. We also study larger $D$ values, where the junction enters the tunneling regime. For the calculation of transport we shall, in the usual way, divide the system into left lead $(L)$, central $(C)$, and right lead $(R)$ regions. The leads are modeled with infinite surfaces, where the fcc [001] axis coincides with the transport direction. We index the regions with the label $\Omega=L, C, R$. As a matrix index, this label also indicates collectively all the orbital indices of the respective region 23$]$.

We assume that the $L$ and $R$ lead potentials are spatially constant and harmonic with angular frequency $\omega$, such that $T_{\omega}=2 \pi / \omega$ is the oscillation period. Thus $\boldsymbol{W}_{\Omega \Omega}(t)=U_{\Omega}(t) \boldsymbol{S}_{\Omega \Omega}$, where $U_{\Omega}(t)=U_{\Omega}^{d c}+U_{\Omega}^{a c} \cos (\omega t)$, and $\Omega=L, R$. The applied dc voltage $V=\left(U_{L}^{d c}-U_{R}^{d c}\right) / e$, where $-e$ is the electron charge, is assumed to be infinitesimal. For the lead-center hoppings we also assume $\boldsymbol{W}_{C \Omega}(t)=U_{\Omega}(t) \boldsymbol{S}_{C \Omega}$, etc. The central potential is assumed to be of the form $\boldsymbol{W}_{C C}(t)=\boldsymbol{W}_{C C}^{d c}+$ $\boldsymbol{W}_{C C}^{a c} \cos (\omega t)$, or $\left[\boldsymbol{W}_{C C}(t)\right]_{i j}=\left[\boldsymbol{S}_{C C}\right]_{i j}\left(U_{i}(t)+U_{j}(t)\right) / 2$ [26]. Here, $U_{i}(t)=U_{i}^{d c}+U_{i}^{a c} \cos (\omega t)$ is the same for all orbitals $i$ on the same atom in region $C$. The actual shape of $U_{i}(t)$ within $C$ should in principle be obtained selfconsistently through the solution of a Poisson equation, such that screening and local field-enhancement effects would be properly accounted for. In this case the appearance of higher harmonics of $\omega$ and phase shifts in $\boldsymbol{W}_{C C}(t)$ would be possible. As this would be computationally very costly, we shall do the following: the dc part $U_{i}^{d c}$ is fixed by a requirement of local charge neutrality in equilibrium [23, 26] and incorporated into $H_{C C}$, while for $U_{i}^{a c}$ we just assume some simple forms. Below we consider two model profiles [see Fig. 1(b)]: $U_{i}^{a c}=\left(U_{L}^{a c}+U_{R}^{a c}\right) / 2$ for all orbitals $i$ in region $C$ (profile A), and one which linearly interpolates between $U_{L}^{a c}$ and $U_{R}^{a c}$ (profile B). For the symmetric junctions that we are considering, symmetric profiles of this type are the most reasonable.

\section{CURRENT FORMULA}

We only consider the time-averaged current, and can thus neglect displacement contributions without losing current conservation [10, 11, 12]. The particle current is 
given in terms of non-equilibrium Green functions by [9]

$$
I=\frac{e}{\hbar} \int_{0}^{T_{\omega}} \frac{d t}{T_{\omega}} \operatorname{Tr}\left[\left(\boldsymbol{G}_{C L}^{<} \circ \boldsymbol{t}_{L C}-\boldsymbol{t}_{C L} \circ \boldsymbol{G}_{L C}^{<}\right)(t, t)\right] .
$$

Here, in the case of the non-orthogonal basis [23]

$$
\boldsymbol{t}_{C L}\left(t, t^{\prime}\right)=\left[\left(\boldsymbol{H}_{C L}+\boldsymbol{W}_{C L}(t)\right)-\boldsymbol{S}_{C L} i \hbar \partial_{t}\right] \hbar \delta\left(t-t^{\prime}\right),
$$

and using the "Langreth rules", $\boldsymbol{G}_{C L}^{<}=\boldsymbol{G}_{C C}^{r} \circ \boldsymbol{t}_{C L} \circ$ $\boldsymbol{g}_{L L}^{<}+\boldsymbol{G}_{C C}^{<} \circ \boldsymbol{t}_{C L} \circ \boldsymbol{g}_{L L}^{a}[9]$. The product $\circ$ is defined by $(\boldsymbol{A} \circ \boldsymbol{B})\left(t, t^{\prime}\right)=\int(d s / \hbar) \boldsymbol{A}(t, s) \boldsymbol{B}\left(s, t^{\prime}\right)$, where $\boldsymbol{A}$ and $\boldsymbol{B}$ are matrices in the spin-orbital basis, over which the trace Tr acts. The Green functions $\boldsymbol{G}_{\Omega \Omega^{\prime}}^{x}$ with $x=r, a, \gtrless$ and $\Omega, \Omega^{\prime}=L, C, R$ are defined as usual [9, 23], and $\boldsymbol{g}_{\Omega \Omega}^{x}$ are the functions for uncoupled leads. The $C C$ component of the full retarded function satisfies

$$
\begin{aligned}
{\left[\boldsymbol{S}_{C C} i \hbar \frac{\partial}{\partial t}-\right.} & \left.\boldsymbol{H}_{C C}-\boldsymbol{W}_{C C}(t)\right] \boldsymbol{G}_{C C}^{r}\left(t, t^{\prime}\right)=\hbar \delta\left(t-t^{\prime}\right) \\
& +\left(\boldsymbol{\Sigma}_{L}^{r} \circ \boldsymbol{G}_{C C}^{r}\right)\left(t, t^{\prime}\right)+\left(\boldsymbol{\Sigma}_{R}^{r} \circ \boldsymbol{G}_{C C}^{r}\right)\left(t, t^{\prime}\right),
\end{aligned}
$$

whereas $\boldsymbol{G}_{C C}^{\gtrless}=\boldsymbol{G}_{C C}^{r} \circ\left(\boldsymbol{\Sigma}_{L}^{\gtrless}+\boldsymbol{\Sigma}_{R}^{\gtrless}\right) \circ \boldsymbol{G}_{C C}^{a}$. Here we defined the "lead self-energies" $\boldsymbol{\Sigma}_{\Omega}^{x}=\boldsymbol{t}_{C \Omega} \circ \boldsymbol{g}_{\Omega \Omega}^{x} \circ \boldsymbol{t}_{\Omega C}$, with $\Omega=L, R$. The solutions of

$$
\left[\boldsymbol{S}_{\Omega \Omega} i \hbar \frac{\partial}{\partial t}-\boldsymbol{H}_{\Omega \Omega}-\boldsymbol{W}_{\Omega \Omega}(t)\right] \boldsymbol{g}_{\Omega \Omega}^{r}\left(t, t^{\prime}\right)=\hbar \delta\left(t-t^{\prime}\right)
$$

lead to self-energies of the form

$$
\boldsymbol{\Sigma}_{\Omega}^{x}\left(t, t^{\prime}\right)=e^{-i \int_{t^{\prime}}^{t} \frac{d s}{\hbar} U_{\Omega}(s)} \int \frac{d \epsilon}{2 \pi} e^{-i \epsilon\left(t-t^{\prime}\right) / \hbar} \boldsymbol{\Sigma}_{\Omega}^{x, e q}(\epsilon) .
$$

The equilibrium self-energies are given by $\boldsymbol{\Sigma}_{\Omega}^{x, e q}(\epsilon)=$ $\boldsymbol{t}_{C \Omega}(\epsilon) \boldsymbol{g}_{\Omega \Omega}^{x, e q}(\epsilon) \boldsymbol{t}_{\Omega C}(\epsilon)$, where the Green functions $\boldsymbol{g}_{\Omega \Omega}^{x, e q}(\epsilon)$ of the infinite surfaces are obtained by a decimation method [23], while $\boldsymbol{t}_{C \Omega}(\epsilon)=\boldsymbol{H}_{C \Omega}-\epsilon \boldsymbol{S}_{C \Omega}$ and $\boldsymbol{t}_{\Omega C}=$ $\left[\boldsymbol{t}_{C \Omega}\right]^{\dagger}$. We also have $\boldsymbol{g}_{\Omega \Omega}^{<, e q}(\epsilon)=-f(\epsilon)\left[\boldsymbol{g}_{\Omega \Omega}^{r, e q}(\epsilon)-\boldsymbol{g}_{\Omega \Omega}^{a, e q}(\epsilon)\right]$ where $f(\epsilon)$ is the Fermi function.

Since the Green functions and self-energies $\boldsymbol{A}\left(t, t^{\prime}\right)$ are all periodic in the time $\left(t+t^{\prime}\right) / 2$, we can simplify the analysis by working in Fourier coordinates. We define the harmonic matrices $\hat{A}(\epsilon)$ with components $[\hat{A}]_{m, n}(\epsilon)=$ $\boldsymbol{A}_{m-n}(\epsilon+(m+n) \hbar \omega / 2)$, where $m, n$ are integers, and

$$
\boldsymbol{A}_{n}(\epsilon)=\int_{0}^{T_{\omega}} \frac{d T}{T_{\omega}} e^{i n \omega T} \int \frac{d \tau}{\hbar} e^{i \epsilon \tau / \hbar} \boldsymbol{A}(T+\tau / 2, T-\tau / 2) .
$$

In this way we find $\hat{G}^{\gtrless}=\hat{G}^{r}\left(\hat{\Sigma}_{L}^{\gtrless}+\hat{\Sigma}_{R}^{\gtrless}\right) \hat{G}^{a}$, while

$$
\left[\hat{G}^{r, a}\right]^{-1}=\left[\hat{\epsilon} S_{C C}-H_{C C}\right] \hat{1}-\hat{W}-\hat{\Sigma}_{L}^{r, a}-\hat{\Sigma}_{R}^{r, a} .
$$

Here $[\hat{\epsilon}]_{m, n}=(\epsilon+m \hbar \omega) \delta_{m, n},[\hat{W}]_{m, n}=\boldsymbol{W}_{C C}^{a c}\left(\delta_{m-1, n}+\right.$ $\left.\delta_{m+1, n}\right) / 2$, and we have dropped the indices $C C$ from the harmonic matrices. The self-energies as well as the scattering rates $\hat{\Gamma}_{\Omega}=i\left(\hat{\Sigma}_{\Omega}^{r}-\hat{\Sigma}_{\Omega}^{a}\right)$ are related to the corresponding equilibrium quantities by

$$
\left[\hat{\Sigma}_{\Omega}^{x}\right]_{m, n}=\sum_{l}\left[\hat{\Sigma}_{\Omega}^{x(l)}\right]_{m, n}, \quad\left[\hat{\Gamma}_{\Omega}\right]_{m, n}=\sum_{l}\left[\hat{\Gamma}_{\Omega}^{(l)}\right]_{m, n}
$$

where we define the components

$$
\left[\hat{\Gamma}_{\Omega}^{(l)}\right]_{m, n}(\epsilon)=J_{m+l}\left(\alpha_{\Omega}\right) J_{n+l}\left(\alpha_{\Omega}\right) \boldsymbol{\Gamma}_{\Omega}^{e q}\left(\epsilon-U_{\Omega}^{d c}-l \hbar \omega\right),
$$

with a similar equation for $\hat{\Sigma}_{\Omega}^{x(l)}(\epsilon)$. Here $\boldsymbol{\Gamma}_{\Omega}^{e q}(\epsilon)=$ $i\left[\boldsymbol{\Sigma}^{r, e q}(\epsilon)-\boldsymbol{\Sigma}^{a, e q}(\epsilon)\right], J_{l}$ are Bessel functions of the first kind, and $\alpha_{\Omega}=U_{\Omega}^{a c} / \hbar \omega$. All of the harmonic matrices satisfy the symmetry $\left[\hat{A}^{(l)}\right]_{m+k, n+k}(\epsilon)=\left[\hat{A}^{(l+k)}\right]_{m, n}(\epsilon+$ $k \hbar \omega)$. Finally, we may note that $\int\left(d t / T_{\omega}\right) \operatorname{Tr}[(\boldsymbol{A} \circ$ $\boldsymbol{B})(t, t)]=\int_{0}^{\hbar \omega}(d \epsilon / 2 \pi) \operatorname{Tr}_{\omega}[\hat{A}(\epsilon) \hat{B}(\epsilon)]$, where $\operatorname{Tr}_{\omega}[\hat{A}(\epsilon)]=$ $\sum_{m} \operatorname{Tr}[\hat{A}]_{m, m}(\epsilon)$. For numerical calculations, the matrices $\hat{A}(\epsilon)$ must be truncated to a few lowest indices $m, n$, but the results converge rapidly with the cutoff.

Using the above definitions and general symmetries like $\hat{G}^{r}-\hat{G}^{a}=\hat{G}^{>}-\hat{G}^{<}$, Eq. (2) yields the dc current

$$
I=\frac{e}{\hbar} \int_{0}^{\hbar \omega} \frac{d \epsilon}{2 \pi} \sum_{k, l} \operatorname{Tr}_{\omega}\left[\hat{G}^{r} \hat{\Gamma}_{R}^{(k)} \hat{G}^{a} \hat{\Gamma}_{L}^{(l)}\right]\left(f_{L}^{(l)}-f_{R}^{(k)}\right),
$$

where $f_{\Omega}^{(k)}(\epsilon)=f\left(\epsilon-U_{\Omega}^{d c}-k \hbar \omega\right)$. In the absence of an ac field this reduces to the standard Landauer-type formula [27]. Although we are not computing $U_{i}(t)$ selfconsistently, Eq. (8) is still gauge invariant in the sense that a spatially constant potential added everywhere has no effect. Thus, the results only depend on $U_{L}(t)-U_{R}(t)$ 11].

\section{RESULTS}

The experimentally accessible quantity which we calculate is the linear conductance $G_{d c}(\omega)=\partial I /\left.\partial V\right|_{V=0}$. In what follows we shall assume zero temperature. If the lead self-energies $\Sigma_{L, R}^{r, e q}$ are furthermore assumed to be energy-independent ("wide-band" approximation), then the full result for potential profile A simplifies to [1]

$$
G_{d c}(\omega)=G_{0} \sum_{l}\left[J_{l}(\alpha / 2)\right]^{2} T^{e q}\left(\epsilon_{F}+l \hbar \omega\right),
$$

where $G_{0}=2 e^{2} / h$ and $T^{e q}(\epsilon)$ is the equilibrium transmission function [28]. We have also defined the parameter $\alpha=\alpha_{L}-\alpha_{R}=\left(U_{L}^{a c}-U_{R}^{a c}\right) / \hbar \omega$, which measures the local intensity of the radiation [2]. Equation (9) describes electrons incident from the different sidebands (after having "absorbed" or "emitted" $l$ photons) being transmitted elastically through the constriction, which mostly determines $T^{e q}(\epsilon)$. In reality, the electric field is nonzero only in the constriction and thus the actual physical transitions must occur there. Note that Eq. (9) should reproduce the results for profile A only in the limit of small $\omega$ and $\alpha$. Still, it works surprisingly well for all the cases presented below.

Let us first discuss the results for the contact regime, which is the subject of our main interest in this paper. In Fig. 2 we show results for a dimer $\mathrm{Al}$ contact, where $\epsilon_{F}$ lies in the $3 p$ band. The upper panel shows 

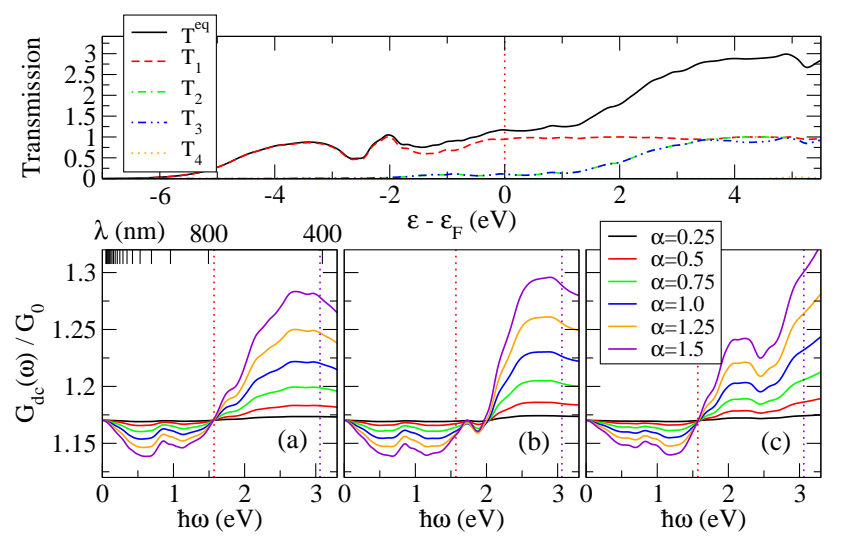

FIG. 2: (Color online) Top panel: Equilibrium transmission $T^{e q}$ and its decomposition into conduction channels $T_{1,2,3,4}$ for an $\mathrm{Al}$ dimer contact. The position of $\epsilon_{F}$ is indicated by a vertical dotted line. Lower panels: Zero-temperature photoconductance for several values of $\alpha$ as a function of frequency $\omega$ using the voltage profile A (a), profile B (b) and Eq. (9) (c). In (b) the wavelengths $\lambda$ with a tick spacing of $400 \mathrm{~nm}$ are shown. The range of visible light is indicated by vertical dotted lines.

the transmission function $T^{e q}(\epsilon)$ and the lower panels show $G_{d c}(\omega)$ for the two voltage profiles as well Eq. (9) for several values of $\alpha$. In the absence of an ac voltage $(\omega=0)$ the conductance is close to $G_{0}$, and is dominated by three conduction channels due to the contribution of $3 s$ and $3 p$ orbitals [20]. At finite $\omega$, the relative change $\delta G_{d c}(\omega)=\left[G_{d c}(\omega)-G_{d c}(\omega=0)\right] / G_{d c}(\omega=0)$ is initially negative, but can then rise to positive values of $\approx 10 \%$ towards visible frequencies. This behavior is similar for both profiles A and B, as well as for Eq. (9). From the latter result, we can interpret our findings in the following appealing way. For $\alpha<1$ only the first sidebands $(l=0, \pm 1)$ contribute to the transport. In this limit, according to Eq. (9) $\delta G_{d c}(\omega)$ measures, roughly speaking, the "second derivative" of $T^{e q}(\epsilon)$ on the scale of $\hbar \omega$ around $\epsilon_{F}$. Thus, for instance, the conductance enhancement in the visible range follows from the transmission increase for electrons promoted above $\epsilon_{F}$ (due to "absorption") overcoming the corresponding decrease for electrons moved below $\epsilon_{F}$ (due to "emission").

Figure 3 shows the corresponding results for $\mathrm{Pt}$. In the absence of radiation the conductance is close to $2.1 G_{0}$ due to the contributions of mainly three conduction channels, which originate from the $6 s$ and $5 d$ orbitals. In this case, and in the contact regime in general for $\mathrm{Pt}$, the effect of the radiation is almost always a significant reduction in conductance. This is understandable, since $\epsilon_{F}$ lies at the edge of the $d$ band, and photon absorption leads to an energy region where fewer open transmission channels are available and $T^{e q}$ is smaller. Note that for low $\omega$, the full results are again well described by Eq. (9). Let us remark that we only compute $G_{d c}(\omega)$ for low enough $\omega$ and $\alpha$, so that the electric fields of the radiation remain reasonable $\left(\lesssim 3 \cdot 10^{9} \mathrm{~V} / \mathrm{m}\right)$.
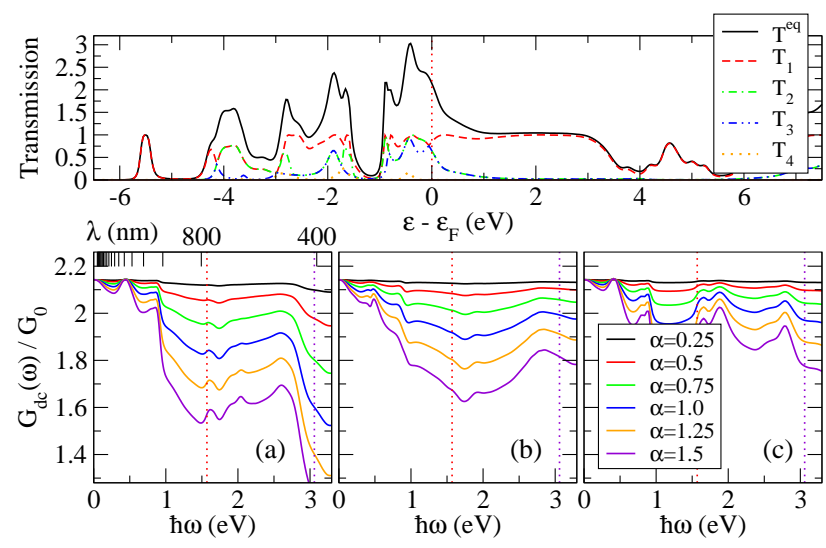

FIG. 3: (Color online) Same as Fig. 2 but for Pt.
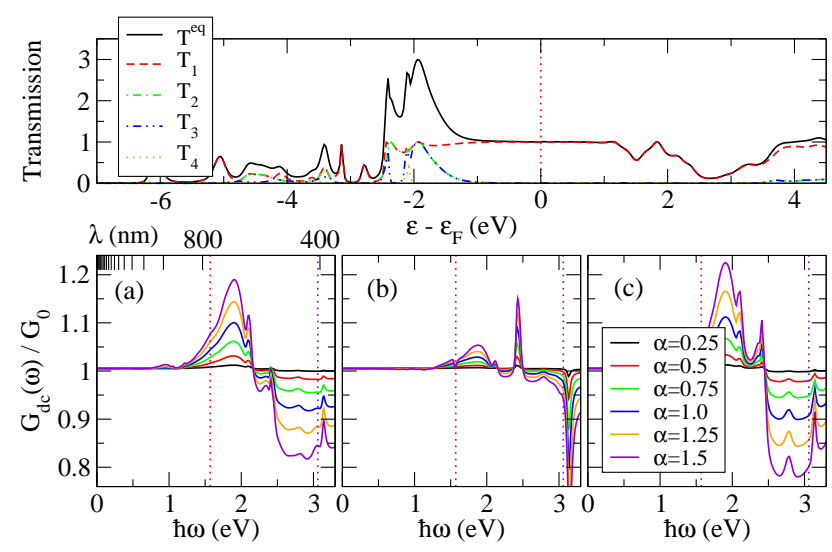

FIG. 4: (Color online) Same as Fig. 2 but for Au.

The results for Au are shown in Fig. 4. As can be seen in the upper panel, the conductance for $\omega=0$ is equal to $1 G_{0}$ with a single open channel arising from the contribution of the $6 s$ orbitals [20]. Moreover, notice that the transmission around $\epsilon_{F}$ is very flat. Due to this flatness, for frequencies up to $\hbar \omega \approx 1 \mathrm{eV}$ the effect of radiation is practically negligible. In the red part of the visible range $(\hbar \omega \lesssim 2 \mathrm{eV})$ we find that $\delta G_{d c}(\omega)>0$ up to a few percent, although this depends rather strongly on the choice of the voltage profile. This increase in the conductance is due to a contribution of the $5 d$ bands located $2 \mathrm{eV}$ below $\epsilon_{F}$, where the number of open transmission channels is higher than at $\epsilon_{F}$. At higher frequencies $\delta G_{d c}(\omega)<0$, as for Pt. We have also studied Au contacts with atomic chains of varying length and the results remain qualitatively similar, although in the case of profile B the amplitude $\delta G_{d c}(\omega)<0$ becomes smaller as the number of chain atoms increases.

When the distance $D$ between the tip atoms of the dimer contact (Fig. 1) is increased, the tunneling regime is approached. Here $G_{d c}(\omega)$ decreases exponentially with increasing $D$, but $\delta G_{d c}(\omega)$ has a tendency to saturate. This is easy to understand, because it may be shown that for very large $D$ the magnitude of the conductance 

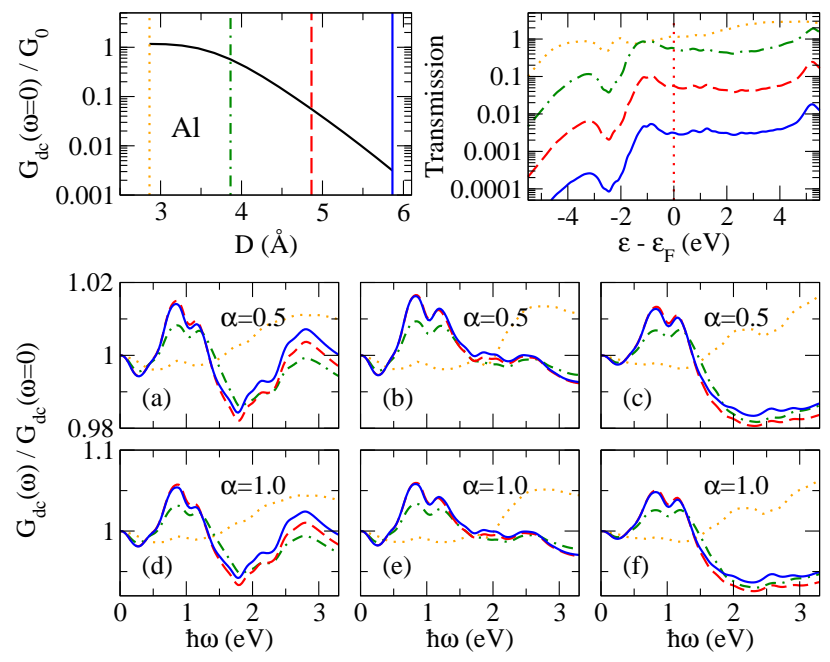

FIG. 5: (Color online) Tunneling limit for Al contacts. Top left panel: zero-frequency conductance $G_{d c}(\omega=0)$ as a function of tip distance $D$. The vertical lines indicate the distances where the examples with corresponding linestyles in the other panels are computed. Top right panel: transmission $T^{e q}(\epsilon)$ for the example distances. Lower panels: the left-hand (a,d), central $(b, e)$, and right-hand $(\mathrm{c}, \mathrm{d})$ panels are for profile A, profile B, and Eq. (9), respectively. The panels (a)-(c) are for $\alpha=0.5$ and (d)-(f) for $\alpha=1.0$.

is approximately determined by the square of the slowestdecaying hopping integral between the tip atoms, which enters the conductance formula as a prefactor. The form of $\delta G_{d c}(\omega)$ can, however, be very different from the contact regime.

An example of the tunneling regime results for $\mathrm{Al}$ is presented in Fig. 5. The top panels illustrate the exponential decay of $G_{d c}(\omega=0)$ and $T^{e q}(\epsilon)$ with $D$, while in the lower panels the quantity $G_{d c}(\omega) / G_{d c}(\omega=0)$ is shown for two values of $\alpha$ and for several distances $D$. The results again look quite similar for the two voltage profiles as well as for Eq. (9). The quantity $\delta G_{d c}$ can obtain both positive and negative values, and its saturation with $D$ is clearly visible. For Pt (see Fig. 6) we find that $G_{d c}(\omega)$ is otherwise flat, but there is a sharp resonance at $\hbar \omega \approx 1 \mathrm{eV}$ where $\delta G_{d c}(\omega)$ can take on positive values of up to a few hundred percent. This is due to resonant transmission through a level formed by the $d$ orbitals of the tip atoms, which can be seen also in the $T^{e q}(\epsilon)$ curves. In the case of $\mathrm{Au}$ there exists a rather similar, but broader positive peak covering the visible range (see Fig. (7). For each metal, we only consider large enough $D$ to see the saturation of $\delta G_{d c}(\omega)$. Indeed, the tightbinding parametrization we are using is based on bulk calculations [24] and is probably not good for very large interatomic distances. Furthermore, the charge neutrality shifts mentioned in Sec. II are strongest for the tip atoms. Therefore the peaks in $G_{d c}(\omega)$ observed for $\mathrm{Pt}$ and $\mathrm{Au}$, for example, should be taken with some reservations. For very large $D$, we would also not expect to see such a good agreement between the results for profiles A
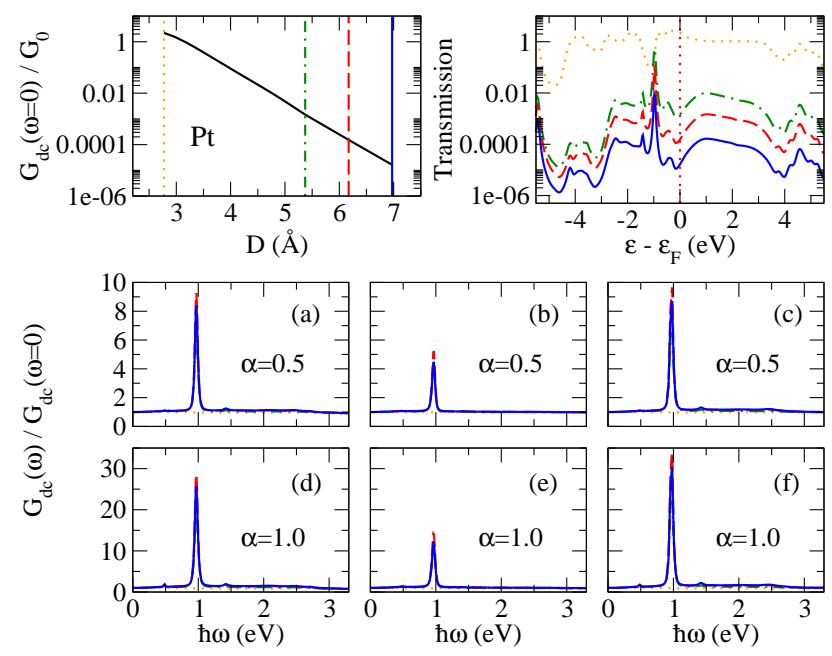

FIG. 6: (Color online) Same as Fig. 5 but for Pt.
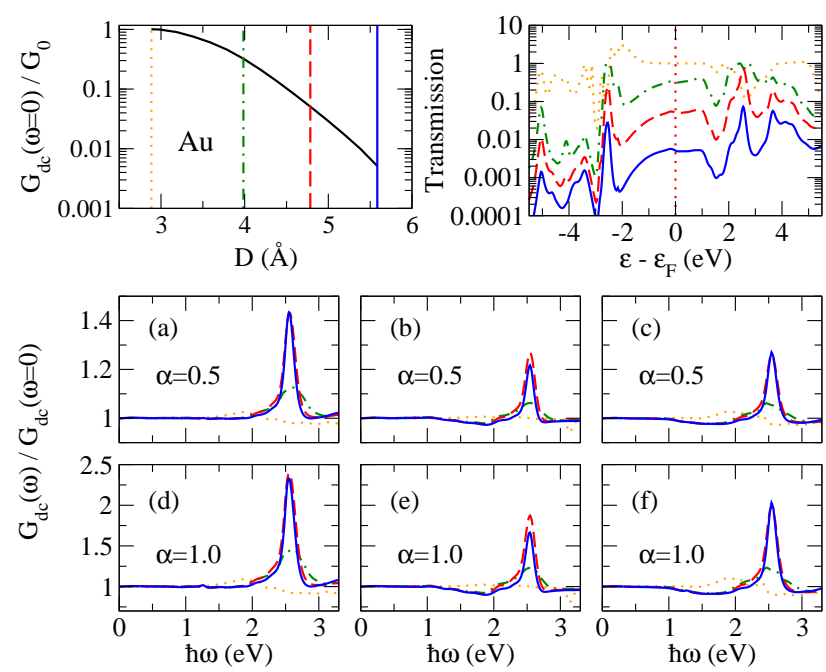

FIG. 7: (Color online) Same as Fig. 5] but for $\mathrm{Au}$.

and $\mathrm{B}$, because the tunneling conductance for profile $\mathrm{B}$ depends on the local densities of states of the tip atoms in a way that cannot be written in the form (9). Nevertheless, our results serve as good illustrations of the different phenomena that may potentially arise in the tunneling regime.

Above we have only shown some example results for very idealized symmetric geometries. In general, the signs and magnitudes of $\delta G_{d c}(\omega)$ depend sensitively on details of the atomic structure, as does $T^{e q}(\epsilon)$. For a more complete analysis one should therefore study also larger contacts and carry out a statistical exploration of geometrical variations along the lines of Ref. 25. Based on Eq. (9), we can still expect that in the limit of severalatoms-wide contacts the relative effect of the ac voltage gradually becomes smaller as $T^{e q}(\epsilon)$ becomes smoother. A direct comparison with the ongoing experiments [19] will be postponed for later. 


\section{DISCUSSION AND CONCLUSIONS}

Some of the assumptions of our model and the effects not taken into account are worth discussing. First, we assume a flat potential in the leads, which requires a complete screening of the electric field. This is well satisfied in metals for frequencies $\omega$ much below the plasma frequency but, as $\omega$ begins to approach the visible range, the screening is weakened. On the other hand, as we have mentioned, the screening in the central region is not treated self-consistently. One of the main concerns here is that local surface-plasmon modes in small geometries tend to have their frequencies close to the visible range, and their excitation can lead to huge field-enhancement effects [5]. Although this is not a problem for our model, one should bear in mind that $\alpha$ is not simply related to the field intensity away from the contact and, following the spirit of the experiments in superconducting contacts [4], it must be understood as an adjustable parameter. Also, at visible frequencies "multiphoton" processes can already cause a photoelectric effect, i.e., excite electrons above the vacuum level, which typically lies $4-6 \mathrm{eV}$ above $\epsilon_{F}$.

Heating accompanies any possible effect arising from absorption of light, which in metals is specially pronounced in the optical range due to the onset of interband transitions. While we may expect that the effect of temperature is just to broaden our results, in practice thermal expansion can play an important role. This is well documented in the STM context [5], where expansion typically brings the tip closer to the sample, thus reducing the tunneling gap width. This results in a strong enhancement of the tunneling current. In the case of atomic wires (i.e., in the contact regime), it is not obvious in which sense and to what degree thermal expansion affects the conductance. Assuming that the expansion simply mimics a mechanical closing process in the STM or break-junction experiments, it can lead to either an increase or a decrease of the conductance, depending on the metal. For instance, for Al contacts, which exhibit raising plateaus upon stretching [29], one would expect a decrease of the conductance due to thermal expansion, as opposed to the effect of the electronic structure in the visible range (see Fig. 2). In this sense, our predictions can be valuable for distinguishing in an experiment (such as Ref. 19) between the contributions of the different effects to the photoconductance.

In conclusion, we have modeled electronic transport in atomic point contacts subjected to external electromagnetic radiation. The radiation has been described by an ac voltage over the contact. Within a non-equilibrium Green function method, we have derived a formula for the dc current in the presence of such an ac drive. Using a tight-binding model, we have applied the method for describing atomic-sized contacts of $\mathrm{Al}, \mathrm{Pt}$, and $\mathrm{Au}$, and have found that the qualitative modification of the dc conductance by the ac voltage can be predicted from the equilibrium transmission function. Depending on the metal, the detailed structure of the contact, and the external frequency, the effect can be either an increase or a decrease in the conductance. At present, experiments are under way to test these predictions [19].

\section{Acknowledgments}

We acknowledge useful discussions with D. Schmidt, E. Scheer, P. Leiderer, R. H. M. Smit, F. Pauly, S. Wohlthat, and M. Häfner. This work was financially supported by the Helmholtz Gemeinschaft (Contract No. VH-NG-029) and by the DFG within the Center for Functional Nanostructures.
[1] J. C. Tucker and M. J. Feldman, Rev. Mod. Phys. 57, 1055 (1985).

[2] P. K. Tien and J. P. Gordon, Phys. Rev. 129, 647 (1963).

[3] J. C. Cuevas, J. Heurich, A. Martín-Rodero, A. Levy Yeyati, and G. Schön, Phys. Rev. Lett. 88, 157001 (2002).

[4] M. Chauvin, P. vom Stein, H. Pothier, P. Joyez, M. E. Huber, D. Esteve, and C. Urbina, Phys. Rev. Lett. 97, 067006 (2006).

[5] S. Grafström, J. Appl. Phys. 91, 1717 (2002).

[6] M. Völcker, W. Krieger, and H. Walther, Phys. Rev. Lett. 66, 1717 (1991).

[7] A. Levy Yeyati and F. Flores, Phys. Rev. B 44, 9020 (1991).

[8] G. Platero and R. Aguado, Phys. Rep. 395, 1 (2004).

[9] A.-P. Jauho, N. S. Wingreen, and Y. Meir, Phys. Rev. B 50, 5528 (1994).

[10] M. Büttiker and T. Christen, Mesoscopic Electron Transport, eds. L. L. Sohn, L. P. Kouwenhoven and G. Schön, Vol 345 of NATO ASI. Series E: Applied Science (Kluwer,
Dordrecht, 1997), p.259.

[11] M. H. Pedersen and M. Büttiker, Phys. Rev. B 58, 12993 (1998).

[12] B. Wang, J. Wang, and H. Guo, Phys. Rev. Lett. 82, 398 (1999).

[13] S. Kohler, J. Lehmann, and P. Hänggi, Phys. Rep. 406, 379 (2005).

[14] W. Zheng, W. Wei, J. Wang, and H. Guo, Phys .Rev. B 61, 13121 (2000).

[15] M. Galperin and A. Nitzan, Phys. Rev. Lett. 95, 206802 (2005).

[16] J. Buker and G. Kirczenow, Phys. Rev. B 66, 245306 (2002).

[17] J. Wu and B. Wang and J. Wang and H. Guo, Phys. Rev. B 72, 195324 (2005).

[18] For a recent review see N. Agraït, A. Levy Yeyati, and J.M. van Ruitenbeek, Phys. Rep. 377, 81 (2003).

[19] D. Guhr, D. Rettinger, J. Boneberg, A. Erbe, P. Leiderer, and E. Scheer, cond-mat/0612117.

[20] E. Scheer, N. Agraït, J. C. Cuevas, A. L. Yeyati, B. 
Ludolph, A. Martin-Rodero, G. R. Bollinger, J. M. van Ruitenbeek, C. Urbina, Nature 394, 154 (1998).

[21] S. Datta and M. P. Anantram Phys. Rev. B 45, 13761 (1992); M. P. Anantram and S. Datta, Phys .Rev. B 51, 7632 (1995).

[22] T. Brandes, Phys. Rev. B 56, 1213 (1997).

[23] J. K. Viljas, J. C. Cuevas, F. Pauly, and M. Häfner, Phys. Rev. B 72, 245415 (2005).

[24] M. J. Mehl and D. A. Papaconstantopoulos, Phys. Rev. B 54, 4519 (1996); D. A. Papaconstantopoulos and M. J. Mehl, J. Phys. Condens. Matter 15, R413 (2003).

[25] M. Dreher, F. Pauly, J. Heurich, J. C. Cuevas, E. Scheer, and P. Nielaba, Phys. Rev. B 72, 075435 (2005); F. Pauly, M. Dreher, J. K. Viljas, M. Häfner, J. C. Cuevas, and P. Nielaba, Phys. Rev. B 74, 235106 (2006).
[26] This choice for representing the potential profile in a nonorthogonal basis is discussed for example in $\mathrm{M}$. Brandbyge, N. Kobayashi, and M. Tsukada, Phys. Rev. B 60, 17064 (1999).

[27] S. Datta, Electronic Transport in Mesoscopic Systems (Cambridge University Press, Cambridge, 1995).

[28] This is given in the usual way by $T^{e q}=\operatorname{Tr}\left[\boldsymbol{t} \boldsymbol{t}^{\dagger}\right]$, where $\boldsymbol{t}=$ $\left(\boldsymbol{\Gamma}_{L}^{e q}\right)^{1 / 2} \boldsymbol{G}_{C C}^{r, e q}\left(\boldsymbol{\Gamma}_{R}^{e q}\right)^{1 / 2}[23,25,[27]$, and the eigenchannel transmissions are defined as the eigenvalues of $\boldsymbol{t} \boldsymbol{t}^{\dagger}$. Due to spin degeneracy, the matrices are now in a basis with only a single spin species.

[29] J. C. Cuevas, A. Levy Yeyati, A. Martín-Rodero, G. R. Bollinger, C. Untiedt, and N. Agraït, Phys. Rev. Lett. 81, 2990 (1998). 